\title{
Modeling and Analysis of Molten Fuel Vaporization and Expansion for a Sodium Fast Reactor Severe Accident
}

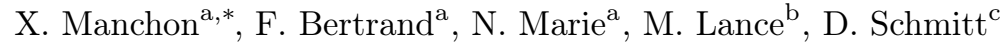 \\ ${ }^{a}$ Commissariat à l'Énergie Atomique et aux Energies Alternatives, Cadarache, F-13108 Saint-Paul-lez-Durance, France \\ ${ }^{b}$ Ecole Centrale de Lyon, 36 av. Guy de Collongue, F-69134 Ecully, France \\ ${ }^{c}$ Électricité de France, EDF Lab Paris-Saclay, 7 boulevard Gaspard Monge, F-91120 Palaiseau, France
}

\begin{abstract}
The safety assessment of Sodium-cooled Fast Reactors (SFR) requires to account for hypothetical severe accidents involving the melting down of the core materials. This paper deals with the modeling of a fuel vaporization transient that might occur in a SFR in case of severe accident. After a nuclear power excursion, some fuel might be molten and vaporized. In this case, the expansion of fuel vapor might generate a mechanical stress on the reactor vessel. Assessing the vessel integrity is of major importance for the reactor design. A fuel vaporization and expansion modeling, which has been simplified using a dimensional analysis, is presented. The modeling is implemented in a tool able to perform fast calculations, of the order of one minute. The vaporized fuel's thermal exchange with the reactor liquid coolant leading to a possible coolant vaporization is simulated by the tool. As the mass of coolant that might be vaporized is a source of uncertainty, two entrainment models are proposed, one based on a Rayleigh-Taylor instability and another one based on a Weber stability criterion. The modeling is validated on other simulations and on experimental results. Parametric calculations are conducted on a reactor case. The impact of the initial molten fuel mass, its initial temperature, critical Weber number and radiative heat transfer are investigated. The non-adiabatic modeling and the adiabatic modeling yield results different by $60 \%$ in certain cases. The tool is shown to be sensitive to the fuel initial temperature, the heat transfer coefficient and the Rayleigh-Taylor wavelength, involving variations that can range to $30 \%$.
\end{abstract}

Keywords: SFR, Core Disruptive Accident, Fast-running tool, Dimensional Analysis, Interface instability.

\section{Highlights}

- Evaluation tool for nuclear fuel vaporization to analyze SFR safety.

- Comparison of adiabatic and non-adiabatic expansions due to sodium entrainment.

- Comparison to the EXCOBULLE tests and to PFBR safety studies is provided.

- Parametric studies on a SFR-like geometry to assess the released energy variability.

\section{Introduction}

A Sodium-cooled Fast Reactor (SFR) of Generation IV (figure 1) is under development in France. The reactor safety analyses have to account for severe accidents, involving the melting down of the reactor's core.

However, severe accidents involve highly uncertain phenomena and parameters. Lack of data accuracy and of understanding of interactions between different physics generate uncertainties. Assessing uncertainties and evaluating safety margins with parametric studies are the main purpose of fast-running tools development. Such assessment relies on the combination of these fast-running tools (Marie et al., 2013, 2016a,b; Droin et al., 2015; Herbreteau et al., 2016) with statistical analysis techniques (Marrel et al., 2015).

*Corresponding Author: xavier.manchon@cea.fr 


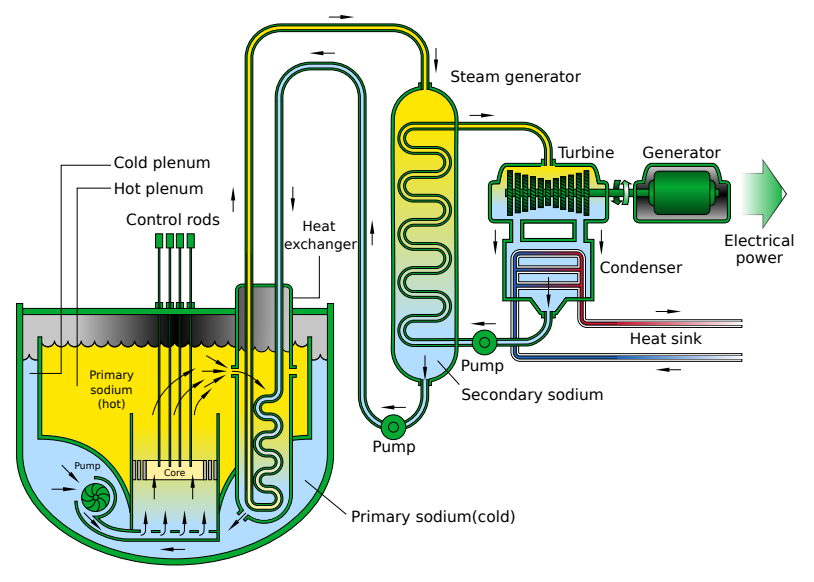

Figure 1: Sodium Fast Reactor concept.

This paper deals with the modeling of the last stage of an energetic Core Disruptive Accident (CDA) and its associated tool development. CDAs were among the first accidents studied for SFRs safety. The term energetic means that the accident leads to a release of mechanical energy that might damage the primary vessel. CDAs lead to the generation of large thermal energies, high enough to heat nuclear fuel far over its saturation temperature, leading to its fast vaporization. This can be achieved when the core of a reactor collapses, leading to a power excursion induced by fissile materials compaction. The mechanical energy release due to materials dispersal after a nuclear excursion caused by the collapse of a SFR core was firstly studied by Bethe and Tait (1956). Even if the Bethe-Tait accident is not always exactly studied as it stood in 1956, the energetic expansion stage is today classical in SFR safety assessment. The most commonly used models for the energetic part of the accident assume that the fuel vaporization creates a high-pressure fuel vapor bubble, which can be several meters large (Epstein et al., 2001). This bubble's expansion loads the primary vessel.

While the fuel vaporization-expansion transient has been widely studied, especially in the adiabatic fuel vapor expansion case, a lack of knowledge remains on some parts of the accident. The thermal exchange between very hot fuel vapor and colder sodium coolant, leading to the coolant vaporization, is notably questionable. Most models (Bertrand et al., 2015; Chellapandi et al., 2010a,b) are built on the assumption that an adiabatic expansion is more energetic than a non-adiabatic one, because the energy lost during the heat transfer will not be converted into mechanical energy. This hypothesis is true while no phase change occurs. But if the heat transfer causes sodium vaporization, the conclusion might change. Indeed, the addition of sodium vapor to the fuel vapor bubble might lead to a pressure bubble increase. On the other hand, the fuel vapor/liquid sodium heat transfer increases the fuel condensation, and therefore might decrease the bubble pressure. This issue of competition between vaporization and condensation was first pointed out by Cho and Epstein (1974), and then studied by Corradini (1978), Reynolds and Berthoud (1981), Berthoud et al. (1984) and Epstein et al. (2001). This heat transfer is thought to be driven by the coolant entrainment inside the fuel vapor, caused by interface hydrodynamic instabilities. These instabilities enhance the heat transfer by mixing materials.

Therefore, the modeling presented in this paper displays several degrees of complexity. Section 2.1 describes the general modeling geometry. Section 2.2 is dedicated to the modeling of an adiabatic expansion. In order to develop a physically reliable tool, the modeling assumptions are justified using dimensional analysis. Section 2.3 is dedicated to the modeling of a non-adiabatic expansion. A Rayleigh-Taylor instability (Taylor, 1950) leading to the entrainement of sodium jets inside the fuel vapor and its consequences on heat transfer are described. The issue of the entrained sodium particles' shape is studied. In section 3, the non-adiabatic modeling of the tool is compared to the EXCOBULLE II experiment performed at CEA by Berthoud et al. (1984). Then the physical tool is compared to FUSTIN, an Indian computation tool 


\begin{tabular}{|c|c|c|c|}
\hline \multicolumn{4}{|c|}{ Nomenclature } \\
\hline \multicolumn{2}{|c|}{ Roman symbols } & $d[-]$ & Droplet \\
\hline$A\left[\mathrm{~m}^{2}\right]$ & Area & $\exp [-]$ & Expansion \\
\hline$B i[-]$ & Biot number & $f[-]$ & Fuel \\
\hline$c_{p}\left[\mathrm{~J} \cdot \mathrm{kg}^{-1} \cdot \mathrm{K}^{-1}\right]$ & $\begin{array}{l}\text { Specific heat capacity at constant } \\
\text { pressure }\end{array}$ & $j[-]$ & Jets or droplets family number \\
\hline$d[m]$ & Diameter & jets $[-]$ & Jets \\
\hline $\mathrm{d}[-]$ & Differential operator & $N a[-]$ & Sodium \\
\hline$E u[-]$ & Euler number & $p[-]$ & Pattern \\
\hline $\operatorname{Fr}[-]$ & Froude number & vap $[-]$ & Vaporization \\
\hline$g\left[\mathrm{~m} \cdot \mathrm{s}^{-2}\right]$ & Gravity acceleration & & \\
\hline$H[\mathrm{~J}]$ & Enthalpy & \multicolumn{2}{|l|}{ Superscripts } \\
\hline$h\left[\mathrm{~J} \mathrm{~kg}^{-1}\right]$ & Specific enthalpy & $C[-]$ & Critical \\
\hline$J a[-]$ & Jakob number & $\operatorname{conv}[-]$ & Convection \\
\hline$K[\mathrm{~J}]$ & Kinetic energy & eff $[-]$ & Effective \\
\hline$k\left[\mathrm{~W} \cdot \mathrm{m}^{-1} \cdot \mathrm{K}^{-1}\right]$ & Thermal conductivity & $l[-]$ & Liquid \\
\hline$L[\mathrm{~m}]$ & Characteristic length & $l v[-]$ & Liquid-vapor phase change \\
\hline$m[\mathrm{~kg}]$ & Mass & $l+v[-]$ & Two-phase liquid-vapor mixture \\
\hline$N u[-]$ & Nusselt number & $M[-]$ & Most unstable \\
\hline$n[-]$ & Jets or droplets number & $\operatorname{mix}[-]$ & Mixing \\
\hline$P e[-]$ & Peclet number & $R T[-]$ & Rayleigh-Taylor \\
\hline $\operatorname{Pr}[-]$ & Prandtl number & $\operatorname{rad}[-]$ & Radiative \\
\hline$p[\mathrm{~Pa}]$ & Pressure & sat $[-]$ & Saturation \\
\hline$Q[\mathrm{~J}]$ & Heat transfer & tot $[-]$ & Total \\
\hline$R[\mathrm{~m}]$ & Radius & $v[-]$ & Vapor \\
\hline$\dot{R}\left[\mathrm{~m} . \mathrm{s}^{-1}\right]$ & Radius time-derivative & $\operatorname{vap}[-]$ & Vaporized \\
\hline$\ddot{R}\left[\mathrm{~m} . \mathrm{s}^{-2}\right]$ & Radius second time-derivative & & \\
\hline $\operatorname{Re}[-]$ & Reynolds number & \multicolumn{2}{|l|}{ Greek symbols } \\
\hline$T[\mathrm{~K}]$ & Temperature & $\Delta[-]$ & Variation \\
\hline$t[\mathrm{~s}]$ & Time & $\delta[-]$ & Infinitesimal variation \\
\hline$V\left[\mathrm{~m}^{3}\right]$ & Volume & $\varepsilon[-]$ & Radiative emissivity \\
\hline$v\left[\mathrm{~m} \cdot \mathrm{s}^{-1}\right]$ & Velocity & $\gamma[-]$ & Specific heat capacities ratio \\
\hline$W[\mathrm{~J}]$ & Work & $\kappa\left[\mathrm{W} \cdot \mathrm{m}^{-2} \cdot \mathrm{K}^{-1}\right]$ & Heat transfer coefficient \\
\hline$W e[-]$ & Weber number & $\lambda[\mathrm{m}]$ & Wavelength \\
\hline$x[-]$ & Vapor quality & $\eta[\mathrm{Pa} . \mathrm{s}]$ & Dynamic viscosity \\
\hline \multirow{2}{*}{\multicolumn{2}{|c|}{ Subscripts }} & $\vec{\nabla}\left[\mathrm{m}^{-1}\right]$ & Gradient operator \\
\hline & & $\rho\left[\mathrm{kg} \cdot \mathrm{m}^{-3}\right]$ & Density \\
\hline$A r[-]$ & Argon & $\nu\left[\mathrm{W} \cdot \mathrm{m}^{2} \cdot \mathrm{K}^{-4}\right]$ & Stefan-Boltzmann constant \\
\hline$b[-]$ & Bubble & $\sigma\left[\mathrm{J} . \mathrm{m}^{-2}\right]$ & Surface tension \\
\hline
\end{tabular}

based on an adiabatic expansion modeling (Chellapandi et al., 2010a). In section 4, parametric calculations investigate the effects of initial molten fuel mass and temperature, break-up and radiative heat transfer.

As a large number of uncertain parameters and phenomena are involved in this transient, fast-running tools are valuable to perform statistical studies. Currently, there is no available fast-running tool to deal with such fuel vaporization transient. The modeling presented in this paper aims to solve this issue. Order of magnitudes are compared in order to identify which phenomena are the most important for the assessment of the vapor expansion's mechanical consequences. This should help to improve SFR's safety by identifying parameters and phenomena of interest that would require experiments or more advanced models. 


\section{Modeling}

\subsection{Main assumptions}

The molten fuel studied is composed by uranium and plutonium oxides. A non-modeled nuclear power excursion is assumed to initiate the fuel melting down and superheating. The vessel's mechanical deformation is not modeled. Only thermal-hydraulics and thermodynamics are thus considered in the paper. The input data are the initial molten fuel mass, which is of the order of several tons, and its temperature, ranging from $4000 \mathrm{~K}$ to $5000 \mathrm{~K}$. These values come from typical severe accident studies (Bertrand et al., 2015). The corresponding fuel saturation pressure is of the order of several MegaPascals (equation (52) in appendix B).

The system considered to model molten fuel expansion consists in four phases distributed in a spherical 1-D geometry, schematized in figure 2. The phases are liquid fuel, fuel vapor, sodium and argon. The liquid fuel is superheated and is cooled down by its own vaporization. The expanding fuel vapor receives a mass flow from the liquid phase and pushes away the sodium phase. The sodium phase represents the reactor coolant, modeled as a liquid piston. The argon phase represents the core cover gas, compressed when the sodium moves.

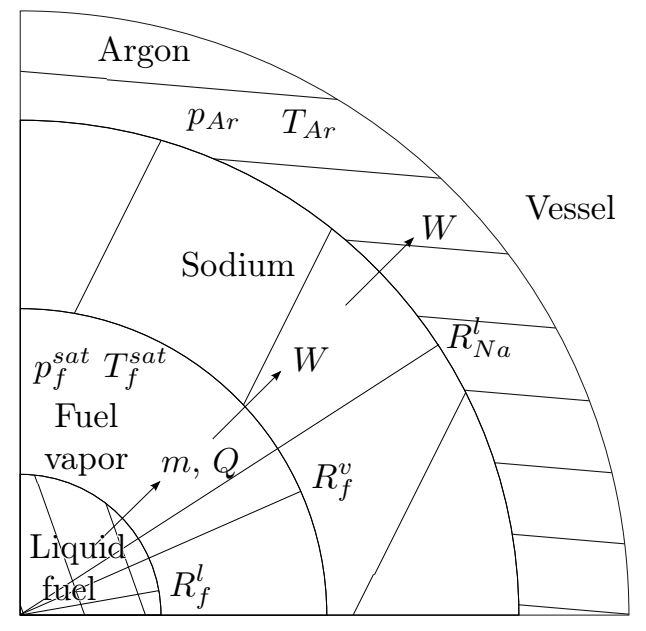

Figure 2: Top-right quarter of the modeled geometry.

The two-phase fuel is supposed to be near its saturation conditions. The sodium flow is assumed to be inviscid and incompressible. The argon compression is supposed to be isentropic. Two kinds of modeling are compared in this paper.

- The so-called adiabatic modeling, described in section 2.2, neglects the heat transfer between the fuel vapor and the liquid sodium. The liquid fuel volume is demonstrated to be constant over the transient. The only energy exchange between the liquid fuel and the fuel vapor results from the heat and mass transfer due to the vaporization process. Under this statement, the fuel vaporization is caused by the vapor pressure decrease, resulting from the fuel expansion.

- The so-called non-adiabatic modeling, described in section 2.3, considers a mass and heat transfer through the fuel vapor/sodium interface, caused by interface instabilities.

\subsection{Adiabatic modeling}

The modeling hypotheses are justified using Dimensional Analysis in sections 2.2.1, 2.2.2 and 2.2.3. Except the heat transfer between the fuel vapor and liquid sodium, the assumptions of the adiabatic and non-adiabatic models are the same. The modeling equations are described in section 2.2.4. Fuel, sodium and argon are respectively named $f, N a$ and $A r$. The materials' state (liquid or vapor) are named $l$ and $v$. 


\subsubsection{Liquid fuel volume variation}

First, the liquid fuel volume's relative variation during the transient is analyzed. Assuming that the liquid specific heat is converted into latent heat, the liquid global enthalpy balance integrated over the transient is

$\rho_{f}^{l} V_{f}^{l} c_{p_{f}}^{l} \Delta T_{f}^{l}=h_{f}^{l v} \Delta m_{f}^{l}=h_{f}^{l v} \rho_{f}^{l} \Delta V_{f}^{l}$,

where $\rho$ is a density, $V$ a volume, $c_{p}$ a specific heat capacity and $h^{l v}$ a phase-change enthalpy. The terms $\Delta T$ and $\Delta m$ are the materials' temperature and mass variations over the transient. Hence the relative liquid fuel volume variation $\Delta V_{f}^{l} / V_{f}^{v}$ is derived. This ratio is typical of a flash-vaporization (Risse, 2012). A system composed of superheated fuel at $4000 \mathrm{~K}$ is considered. The fuel saturation temperature at atmospheric pressure is around $3500 \mathrm{~K}$ (see equation (52) in appendix B). The volume relative variation's absolute value is thus around $16 \%$, which corresponds to a liquid fuel radius relative variation of $5 \%$. The liquid fuel geometry variations may thereby be neglected.

\subsubsection{Mechanical phenomena}

Next Dimensional Analysis step aims at comparing the effects of dynamic viscosity $\eta$, gravity $g$ and surface tension $\sigma$ with the gradient of pressure $p$ applied to the sodium phase. The usual numbers to characterize these phenomena in a flow are the Euler $(E u)$, Reynolds $(R e)$, Froude $(F r)$ and Weber $(W e)$ numbers

$$
\begin{aligned}
& E u=\frac{p_{f}^{v}-p_{A r}}{\rho_{N a}^{l}\left(\dot{R}_{f}^{v}\right)^{2}}, \\
& R e=\frac{\rho_{N a}^{l} \dot{R}_{f}^{v} L_{R e}}{\eta_{N a}^{l}}, \\
& F r=\frac{\dot{R}_{f}^{v}}{\sqrt{g L_{F r}}}, \\
& W e=\frac{\rho_{N a}^{l}\left(\dot{R}_{f}^{v}\right)^{2} L_{W e}}{\sigma_{N a}},
\end{aligned}
$$

where $\dot{R}_{f}^{v}$ is the speed of the vapor/sodium interface, namely the vapor expansion speed, and $L$ is a characteristic spatial length depending on the phenomenon studied.

The initial pressure difference $p_{f}^{v}-p_{A r}$ should be compared to the other parameters. Moreover, the bubble expansion speed $\dot{R}_{f}^{v}$ is unknown. This parameter is discarded by combining the dimensionless groups of equation (2) into new dimensionless groups. The Euler number and squared Reynolds number product is

$$
E u R e^{2}=\frac{p_{f}^{v}-p_{A r}}{\left(\eta_{N a}^{l}\right)^{2} /\left(\rho_{N a}^{l} L_{R e}^{2}\right)} .
$$

This number is the ratio between the total hydraulic head and the viscous head loss. It is sometimes named Bejan number (Petrescu, 1994). The characteristic length $L_{R e}$ is the radial dimension of the sodium volume, which is close to the reactor size, i.e. $7 \mathrm{~m}$. This number is near $10^{24}$, so viscous stress is negligible compared to the pressure difference. Similarly, the product of the Froude and Euler numbers is

$E u F r^{2}=\frac{p_{f}^{v}-p_{A r}}{\rho_{N a}^{l} g L_{F r}}$.

This number compares the total pressure difference versus the gravity pressure drop. The characteristic length $L_{F r}$ is the sodium radius, on which the pressure difference is applied, close to $7 \mathrm{~m}$. Its value is 
around $10^{2}$, which allows to neglect gravity regarding the pressure difference. The product of the Reynolds and Weber numbers is

$E u W e=\frac{\left(p_{f}^{v}-p_{A r}\right) L_{W e}}{\sigma_{N a}}$.

This last number emphasizes interface stability considerations, so the characteristic length $L_{W e}$ is not the reactor radius but rather the vapor/sodium interface radius, which is close to $1 \mathrm{~m}$. This number's value is around $10^{7}$, so the surface tension effect is negligible compared to the pressure difference.

The pressure difference between fuel vapor and argon phases is therefore be the only significant parameter.

\subsubsection{Expansion and vaporization characteristic times}

The vaporization and expansion characteristic times are compared in order to determine whether one of these two processes limits the transient. The analysis is done on two independent virtual systems, one involving only mechanical phenomena (expansion), the other involving only thermal phenomena (vaporization).

In order to determine the expansion characteristic time, the sodium phase is considered. Considering only the pressure influence, the sodium momentum equation reads

$\rho_{N a}^{l} \frac{\mathrm{d} \vec{v}_{N a}}{\mathrm{~d} t}=-\vec{\nabla} p$,

where $v_{N a}$ is the flow velocity in the sodium phase. The approximations of vectors $\mathrm{d} \vec{v}_{N a} / \mathrm{d} t$ and $\vec{\nabla} p$ to scalars $\Delta R_{f}^{v} / t_{\text {exp }}$ and $\left(p_{f}^{v}-p_{A r}\right) / \Delta R_{f}^{v}$, where $t_{\text {exp }}$ is the characteristic expansion time and $\Delta R_{f}^{v}$ the bubble radius variation, allow to write

$t_{\exp }=\Delta R_{f}^{v} \sqrt{\frac{\rho_{N a}^{l}}{p_{f}^{v}-p_{A r}}}$.

The maximum volume that can be occupied by the fuel vapor is the argon volume, in case of a complete argon volume closure. This volume gives an estimation for $\Delta R_{f}^{v}$. The argon volume being around $196 \mathrm{~m}^{3}$, an upper value for $\Delta R_{f}^{v}$ is $3.6 \mathrm{~m}$, which implies that $t_{\exp } \approx 0.1 \mathrm{~s}$.

The liquid fuel superheat is again considered to be equal to $500 \mathrm{~K}$ (section 2.2.1). A thermal balance over the liquid fuel yields the characteristic vaporization time $t_{\text {vap }}$, namely

$\kappa A \Delta T_{f}^{l} t_{\mathrm{vap}}=h_{f}^{l v} \Delta m_{f}^{v}$,

where $\Delta m_{f}^{v}$ is the vaporized fuel mass, $A$ is the heat exchange area, $\Delta T_{f}^{l}$ is the liquid superheat and $\kappa$ is the heat transfer coefficient.

In order to evaluate $A$, the vaporization process is assumed to take place as nucleate boiling. This assumption is justified by the high liquid fuel superheat, this transient being analogous to a boiling liquid explosive vaporization (Pinhasi et al., 2007). The fuel vaporization is then described by the birth and the growth of small bubbles inside the liquid fuel bath (schematized in figure 3 ). In this case, $A$ corresponds to the sum of the bubbles' areas. The number of bubbles in the liquid is equal to $\Delta V_{f}^{v} / V_{b}$ where $V_{b}$ is the volume of one bubble. If $d_{b}$ is the mean bubbles' diameter, then

$A=\frac{6 \Delta V_{f}^{v}}{d_{b}}$.

In order to evaluate $A$, it is necessary to evaluate the diameter of vapor bubbles. An upper bound is the maximum bubble diameter determined by the hydrodynamic theory (Zuber, 1959)

$d_{b}=\pi \sqrt{\frac{3 \sigma_{f}}{g\left(\rho_{f}^{l}-\rho_{f}^{v}\right)}}$, 


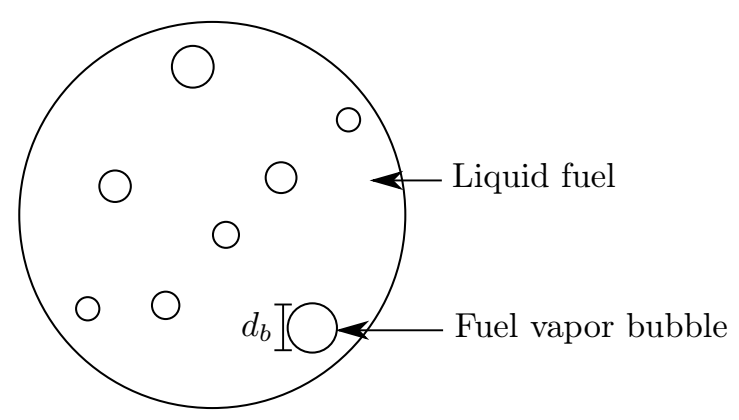

Figure 3: Homogeneous nucleate boiling inside the molten fuel.

which yields $d_{b}=2 \mathrm{~cm}$.

The heat transfer coefficient $\kappa$ is evaluated with a correlation on nucleate boiling determined by Labuntzov et al. (1964), expressing the Nusselt number $N u=\kappa d_{b} / k_{f}^{l}$ and Jakob number $J a=\left(\rho_{f}^{l} c_{p, f}^{l} \Delta T_{f}^{l}\right) /\left(\rho_{f}^{v} h_{f}^{l v}\right)$ as

$N u=\frac{12 J a}{\pi}\left(1+\frac{1}{2}\left(\frac{\pi}{6 J a}\right)^{\frac{2}{3}}+\frac{\pi}{6 J a}\right) \approx \frac{12 J a}{\pi}$.

The Jakob number $J a$ is of the order of 300, which allows the approximation of equation (11). With equations (9) and (11), equation (8) yields

$t_{\mathrm{vap}}=\frac{\pi d_{b}^{2}\left(\rho_{f}^{v}\right)^{2}\left(h_{f}^{l v}\right)^{2}}{72\left(\Delta T_{f}^{l}\right)^{2} \rho_{f}^{l} c_{p, f}^{l} k_{f}^{l}}$.

This analysis yields a vaporization characteristic time is around $1.10^{-3} \mathrm{~s}$.

The fuel vaporization depends on the fuel saturation pressure, which is limited by the fuel expansion that drives the pressure evolution. However, the two characteristic times calculated above show that thermal equilibrium is reached much faster that mechanical equilibrium. Therefore, the fuel can be considered saturated during all the expansion, and the bubble pressure is the fuel saturation pressure.

\subsubsection{Adiabatic modeling differential equations}

The differential equations demonstrations are given in appendix A. To solve the adiabatic expansion, the knowledge of four variables is required :

- the radius of vapor/sodium interface $R_{f}^{v}$, which yields the interface positions along the transient;

- the speed of vapor/sodium interface $\dot{R}_{f}^{v}$, which yields the bubble expansion time;

- the fuel temperature $T_{f}$, which drives the vapor pressure;

- the fuel vapor quality $x$.

The evolution of the vapor/sodium interface radius $R_{f}^{v}$ is simply given by its time derivative $\dot{R}_{f}^{v}$.

The Rayleigh equation (13) (Brennen, 1995), which is the typical momentum equation of a gas bubble inside an incompressible fluid, expresses the acceleration of the vapor/sodium interface. This equation is obtained by spatially integrating the Euler equation over the sodium phase, from the fuel/sodium interface $R_{f}^{v}$ to the sodium/argon interface $R_{\mathrm{Na}}^{l}$, yielding

$$
\frac{\mathrm{d} \dot{R}_{f}^{v}}{\mathrm{~d} t}=\frac{\frac{p_{f}^{v}-p_{A r}}{\rho_{N a}^{l}}-\left(\frac{3}{2}+\frac{1}{2}\left(\frac{R_{f}^{v}}{R_{N a}^{l}}\right)^{4}-\frac{2 R_{f}^{v}}{R_{N a}^{l}}\right)\left(\dot{R}_{f}^{v}\right)^{2}}{R_{f}^{v}-\frac{\left(R_{f}^{v}\right)^{2}}{R_{N a}^{l}}},
$$


where the argon pressure evolution is assumed to follow the isentropic compression law

$p_{A r}=p_{A r}(t=0)\left(\frac{V_{A r}(t=0)}{V_{A r}}\right)^{\gamma}$.

The fuel temperature time derivative is obtained from the balance equation of two-phase fuel enthalpy. This approach is similar to Cho et al. (1972)'s modeling. The two-phase fuel enthalpy $h_{f}^{l+v}$ is written as a function of the fuel vapor quality $x=m_{f}^{v} / m_{f}^{l+v}$ (Delhaye, 2008)

$h_{f}^{l+v}=x h_{f}^{v}+(1-x) h_{f}^{l}$.

Some developments from equation (15), using the global enthalpy balance, lead to

$m_{f}^{l+v}\left\{\left(x \frac{\mathrm{d} h_{f}^{v}}{\mathrm{~d} T_{f}}+(1-x) \frac{\mathrm{d} h_{f}^{l}}{\mathrm{~d} T_{f}}-\frac{V_{f}^{l+v}}{m_{f}^{l+v}} \frac{\mathrm{d} p_{f}}{\mathrm{~d} T_{f}}\right) \frac{\mathrm{d} T_{f}}{\mathrm{~d} t}+h_{f}^{l v} \frac{\mathrm{d} x}{\mathrm{~d} t}\right\}=0$.

In equation (16), the fuel volume $V_{f}^{l+v}$ is expressed as a function of $R_{f}^{v}$. To express the fuel vapor quality $x$, a fourth equation is derived from the two-phase fuel mass

$m_{f}^{l+v}\left(\frac{x}{\rho_{f}^{v}}+\frac{1-x}{\rho_{f}^{l}}\right)=V_{f}^{l+v}$,

and its derivative

$$
\left(x \frac{\mathrm{d}\left(1 / \rho_{f}^{v}\right)}{\mathrm{d} T_{f}}+(1-x) \frac{\mathrm{d}\left(1 / \rho_{f}^{l}\right)}{\mathrm{d} T_{f}}\right) \frac{\mathrm{d} T_{f}}{\mathrm{~d} t}+\left(\frac{1}{\rho_{f}^{v}}-\frac{1}{\rho_{f}^{l}}\right) \frac{\mathrm{d} x}{\mathrm{~d} t}=\frac{1}{m_{f}^{l+v}} \frac{\mathrm{d} V_{f}^{l+v}}{\mathrm{~d} t} .
$$

The equations given below are solved with the materials' equations of state and thermophysical correlations, described in appendix B. The fuel enthalpies and heat capacities are expressed by correlations given by the IAEA (2008). Fuel vapor density's variations with temperature in equation (18) are evaluated using correlations from the SIMMER code (Morita and Fischer, 1998; Morita et al., 1998), which is the current best-estimate numerical tool for SFR severe accidents calculations.

\subsection{Non-adiabatic modeling}

Adiabatic expansion models were usually used to model fuel expansion transients, because it was commonly assumed that adiabatic expansions yield larger mechanical work than non-adiabatic ones. This might not be the case when phase change and pressure variations occur, because they have a direct impact on work. As it was shown experimentally and theoretically (Taylor, 1950; Lewis, 1950), a heavy fluid accelerated by a lighter fluid is unstable, causing Rayleigh-Taylor instabilities. These instabilities entrain sodium jets and droplets inside the fuel vapor. In this case, in order to assess this entrained sodium heat transfer and vaporization, a model accounting for sodium entrainment is proposed. Its main goal lies in the determination of the heat transfer area $A$ and the entrained sodium fragments' diameter $d$. As the fragmentation process is particularly complicated to deal with, several models are proposed. Their respective results are compared in section 4 .

\subsubsection{Rayleigh-Taylor instability}

The instability occurring at the vapor/sodium interface is supposed to be a Rayleigh-Taylor instability. The description of this instability can be found in Ishii and Hibiki (2011). To model this instability, the spherical interface is approximated as a plane two-dimensional interface whose acceleration is $\ddot{R}_{f}^{v}$ (schematized in Figure 4). The sodium jets of same characteristics are gathered into families, numbered $j$, and 
similarly treated. An interface sinusoidal perturbation of wavelength $\lambda$ is studied. The cutoff wavelength, above which the perturbation becomes unstable and starts to grow, is computed at time $t_{j}$ by

$\lambda_{R T, j}^{C}=2 \pi \sqrt{\frac{\sigma_{N a}}{\left(\rho_{N a}^{l}-\rho_{f}^{v}\left(t_{j}\right)\right) \ddot{R}_{f}^{v}\left(t_{j}\right)}}$,

and the most unstable wavelength is

$\lambda_{R T, j}^{M}=\sqrt{3} \lambda_{R T, j}^{C}$.

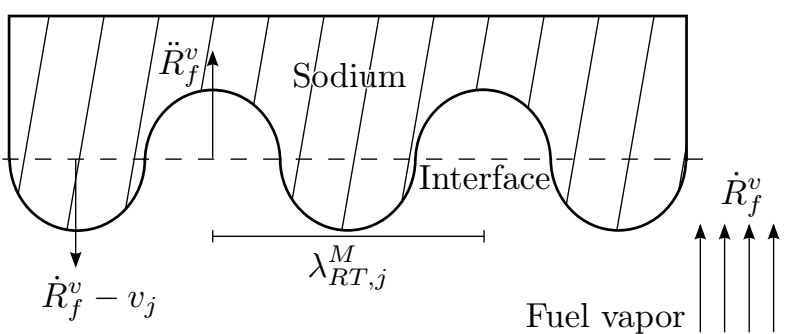

Figure 4: Plane Rayleigh-Taylor instability at fuel vapor/sodium interface

It is assumed that the perturbations developed are only the most unstable ones. Therefore the jets of the $j^{\text {th }}$ family are considered distant of $\lambda_{R T, j}^{M}$ from each other. Once the instability is described, the number of jets entrained at the interface is required. It was shown theoretically by Jacobs and Catton (1988) and then experimentally by Fermigier et al. (1992) that a three-dimensional Rayleigh-Taylor instability will preferentially take the form of a hexagonal pattern. Considering that these hexagons' side length is equal to $\lambda_{R T, j}^{M}$, the number of jets is therefore $n_{\text {jets }, j}=4 \pi\left(R_{f}^{v}\right)^{2} /\left(\sqrt{3}\left(\lambda_{R T, j}^{M}\right)^{2} / 2\right)$.

The perturbations growth rate is required to compute the entrained sodium mass. An experiment conducted by Lewis (1950) showed that a Rayleigh-Taylor jet reaches an asymptotic speed correlated by

$v_{j}=\mathcal{K} \sqrt{\lambda_{R T, j}^{C} \ddot{R}_{f}^{v}}$

where $\mathcal{K}=4.65$ was found experimentally by Corradini et al. (1980). This correlation gives the relative velocity between the entering sodium jets and the fuel vapor.

It is then required to evaluate the length of the jet when it breaks up and the size of the droplets generated by its fragmentation. Fragmentation is a poorly known phenomenon (Villermaux, 2007). Thus, two fragmentation models are proposed in sections 2.3.2 and 2.3.3.

\subsubsection{Rayleigh-Taylor break-up}

This modeling consists in a pure Rayleigh-Taylor fragmentation: the jets are fragmented into spheres whose diameters' are equal to $\lambda_{R T, j}^{M} / 2$. Sharp (1984) indicates that instabilities leading to the breakup of a jet occur after the jet has reached an axial length of the order of $\lambda_{R T, j}^{M}$.

Thus the life-cycle of the $j^{\text {th }}$ family, presented in figure 5 , is the following.

- At time $t_{j}=0$, the $j^{\text {th }}$ jets' family starts to grow at the wavelength $\lambda_{R T, j}^{M}$, evaluated using equation (20). Their growth speed is equal to $v_{j}$ given by equation (21). These jets' parameters are calculated using interface acceleration and materials' densities evaluated at $t_{j}=0 \mathrm{~s}$.

- At time $t_{j+1}=\lambda_{R T, j}^{M} / v_{j}$, when the jets' axial length reaches $\lambda_{R T, j}^{M}$, the jets' fragmentation occurs. Each jet is fragmented into spherical droplets of diameter $d_{j}=\lambda_{R T, j}^{M} / 2$. The jets volume are approximated as cylinders of base $\lambda_{R T, j}^{M} / 2$, so the total entrained volume of family $j$ is

$$
V_{j}=n_{\mathrm{jets}, j} \pi \frac{\left(\lambda_{R T, j}^{M}\right)^{3}}{16} .
$$




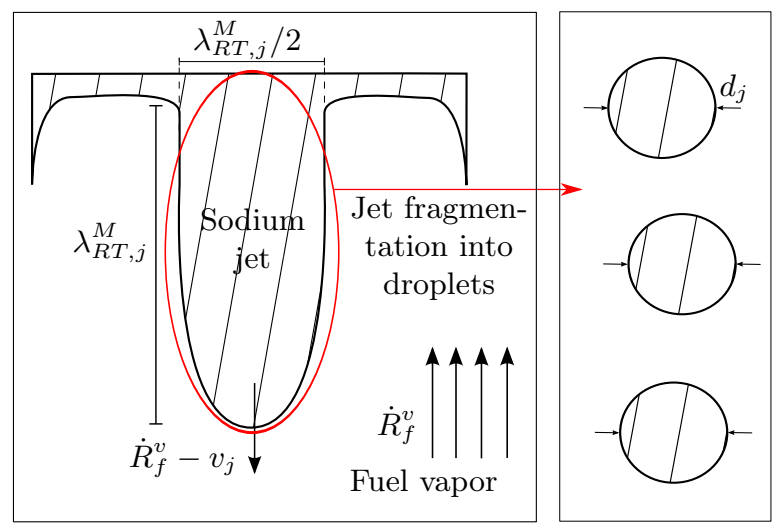

Figure 5: Rayleigh-Taylor break-up

The corresponding number of droplets is $n_{d, j}=V_{j} /\left(4 \pi d_{j}^{3} / 3\right)$. The total heat transfer area $A_{j}$ associated to family $j$ is

$A_{j}=\frac{6 V_{j}}{d_{j}}$.

- At time $t_{j+1}>\lambda_{R T, j}^{M} / v_{j}$, the $j^{\text {th }}$ jets family are dislocated, and therefore the $(j+1)^{\text {th }}$ jets family starts to grow. The cycle repeats until the interface acceleration $\ddot{R}_{f}^{v}$ becomes negative, when the Rayleigh-Taylor instability stops.

\subsubsection{Weber stability criterion}

The analysis conducted in section 2.3.2 postulates that the jets breakup is driven by a Rayleigh-Taylor instability, yielding particles sized of the Rayleigh-Taylor wavelength. Yet, it should be pointed out that the produced droplets might be unstable. A secondary fragmentation might occur. For the present study, the droplet's Weber number is expressed as

$W e_{j}=\frac{\rho_{f}^{v} v_{j}^{2} d_{j}}{\sigma_{N a}}$.

The critical Weber number is classically taken as $W e^{C}=12$ (Kolev, 2012). If the Weber number $W e$ defined by equation (24) is higher than $W e^{C}$, the droplets' diameter $d_{j}$ is redefined as

$d_{j}=\frac{\sigma_{N a} W e^{C}}{\rho_{f}^{v} v_{j}^{2}}$.

\subsubsection{Heat transfer and phase change equations}

In the present section, a method to compute the heat transfer from the fuel vapor to the entrained liquid sodium is proposed.

The fuel/sodium heat transfer takes into account radiative and convective heat transfer models. Regarding radiation, a simple grey-body radiative law (Bergman et al., 2012) is implemented and the sodium emissivity is varied. Their relative velocity through the fuel vapor is $v_{j}$. The $j^{\text {th }}$ sodium droplets family's temperature is $T_{j}$. The convective heat transfer coefficient from the fuel vapor to a sodium droplet is noted $\kappa_{j}$. It is evaluated with the Lee and Riley (1966) correlation, established for water condensation around a sphere, that gives a relation between the Nusselt $(N u)$, Reynolds $(R e)$ and Prandtl $(P r)$ numbers

$N u=\frac{\kappa_{j} d_{j}}{k_{f}^{v}}=2+0.74 R e^{0.5} \operatorname{Pr}^{0.33}$. 
The convective heat transfer rate from the fuel vapor to all the droplets of the $j^{\text {th }}$ family during a time lapse $\mathrm{d} t$ is

$$
\frac{\delta Q_{j}^{c o n v}}{\mathrm{~d} t}=\kappa_{j} A_{j}\left(T_{f}^{v}-T_{j}\right),
$$

where $A_{j}$ is the sum of the droplets areas as defined in sections 2.3.2 and 2.3.3. The sodium is assumed to be a grey-body and the molten fuel a black-body. When the radiative heat transfer rate is modeled, it is computed by

$$
\frac{\delta Q_{j}^{r a d}}{\mathrm{~d} t}=A_{j} \nu \varepsilon_{N a}\left(\left(T_{f}^{l}\right)^{4}-T_{j}^{4}\right),
$$

where $\nu$ and $\varepsilon_{N a}$ are the Stefan-Boltzmann radiation constant and the sodium emissivity. The mass of family $j$ is $m_{j}=n_{d, j} \rho_{N a}^{l} \pi d_{j}^{3} / 6$. Each droplet temperature change is calculated by

$\frac{\mathrm{d} T_{j}}{\mathrm{~d} t}=\frac{1}{m_{j} c_{p, N a}^{l}}\left(\frac{\delta Q_{j}^{c o n v}}{\mathrm{~d} t}+\frac{\delta Q_{j}^{r a d}}{\mathrm{~d} t}\right)$,

where $n_{d, j}$ is the number of droplets defined in section 2.3.2 or section 2.3.3, depending upon the model. Writing equation (29) requires that the sodium droplet temperature profile is flat. This is justified in appendix A.

If $T_{j}(t+\mathrm{d} t)$ is greater than $T_{N a}^{s a t}$, some liquid sodium is vaporized. The vaporized sodium mass $m_{N a}^{v a p}$ is computed by

$m_{N a}^{v a p}=\sum_{j} \frac{\delta Q_{j}^{c o n v}+\delta Q_{j}^{r a d}-m_{j} c_{p, N a}^{l}\left(T_{N a}^{s a t}-T_{j}(t)\right)}{h_{N a}^{l v}}$.

The droplets' sizes and areas are reduced correspondingly to the droplets mass loss caused by their vaporization. Typically, the sodium saturation pressure is close to $1500 \mathrm{~K}$ at $2 \mathrm{MPa}$. The sodium vapor is thus relatively cold compared to the fuel vapor (more than $4000 \mathrm{~K}$ ). It is assumed that the sodium and fuel vapors are instantaneously thermally mixed. Assuming that the vaporized sodium mass is low versus the fuel vapor mass, the mixing heat transfer rate is computed by

$\frac{\delta Q^{m i x}}{\mathrm{~d} t}=\frac{\mathrm{d} m_{N a}^{v a p}}{\mathrm{~d} t} \int_{T=T_{N a}^{s a t}}^{T=T_{f}^{v}} c_{p, N a}^{v} \mathrm{~d} T$.

A total heat transfer is thus computed

$\frac{\delta Q_{j}^{t o t}}{\mathrm{~d} t}=\frac{\delta Q_{j}^{\text {conv }}}{\mathrm{d} t}+\frac{\delta Q_{j}^{m i x}}{\mathrm{~d} t}+\frac{\delta Q_{j}^{\text {rad }}}{\mathrm{d} t}$.

The sodium vaporization leads to a change of the bubble pressure $p_{b}$. The bubble pressure $p_{b}$ is the sum of the fuel and sodium vapors' partial pressures. The sodium partial pressure $p_{N a}$ is computed with a modified Redlich-Kwong equation (given in appendix B). The fuel vapor partial pressure is therefore equal to the fuel saturation pressure at temperature $T_{f}^{v}$.

\subsubsection{Non-adiabatic modeling differential equations}

The adiabatic modeling's equations written in section 2.2.4 change slightly for the non-adiabatic modeling (appendix A). The acceleration equation (13) becomes

$$
\frac{\mathrm{d} \dot{R}_{f}^{v}}{\mathrm{~d} t}=\frac{\frac{p_{b}-p_{A r}}{\rho_{N a}^{l}}-\left(\frac{3}{2}+\frac{1}{2}\left(\frac{R_{f}^{v}}{R_{N a}^{l}}\right)^{4}-\frac{2 R_{f}^{v}}{R_{N a}^{l}}\right)\left(\dot{R}_{f}^{v}\right)^{2}}{R_{f}^{v}-\frac{\left(R_{f}^{v}\right)^{2}}{R_{N a}^{l}}},
$$


and the enthalpy equation (16) becomes

$m_{f}^{l+v}\left\{\left(x \frac{\mathrm{d} h_{f}^{v}}{\mathrm{~d} T_{f}}+(1-x) \frac{\mathrm{d} h_{f}^{l}}{\mathrm{~d} T_{f}}-\frac{V_{f}^{l+v}}{m_{f}^{l+v}} \frac{\mathrm{d} p_{f}^{s a t}}{\mathrm{~d} T_{f}}\right) \frac{\mathrm{d} T_{f}}{\mathrm{~d} t}+h_{f}^{l v} \frac{\mathrm{d} x}{\mathrm{~d} t}\right\}=-\frac{\delta Q^{t o t}}{\mathrm{~d} t}$,

where $\delta Q^{t o t}$ is the sum of the $\delta Q_{j}^{t o t}$ defined by equation (32). Equation (18) is unchanged.

\section{Physical tool numerical and experimental comparisons}

\subsection{Non-adiabatic modeling comparison to the EXCOBULLE II tests}

The EXCOBULLE II tests were achieved at the Nuclear Research Center of Grenoble (France) by Berthoud et al. (1984). These tests were dedicated to the study of the growth and collapse of a superheated two-phase water bubble in a cold liquid water tank at $20{ }^{\circ} \mathrm{C}$. The initial superheated water source is a glass sphere containing pressurized liquid water. This water is heated in ranges of $160{ }^{\circ} \mathrm{C}$ to $280{ }^{\circ} \mathrm{C}$, the corresponding saturation pressure ranging from 1.2 MPa to 6.4 MPa. The sphere, once broken by two long pistons, releases a two-phase steam bubble that rapidly expands and mixes with the liquid coolant. Measurements of the two-phase bubble radius versus time is performed with a high-frequency camera with an estimated uncertainty of $15 \%$.

These tests are the closest experiment to the present modeling. They mostly focus on thermal interactions and mixing due to hydrodynamic instabilities, so comparing the physical tool to this experiment is valuable.

As the EXCOBULLE II tests were done with water, the thermo-physical laws of liquid water and steam have been implemented in the physical tool. The water thermo-physical properties come from Wagner and Pruß (2002). The water tank was open in the EXCOBULLE II tests, so the cover gas pressure is kept to $0.12 \mathrm{MPa}$ in the physical tool calculations. The radiative heat transfer is not modeled for this validation because of the low temperature difference in the tests. The calculations with the Rayleigh-Taylor and Weber fragmentation criteria described in sections 2.2 and 2.3 yield the same result in this case because the experimental transient is not violent enough to further fragment Rayleigh-Taylor-sized water droplets.

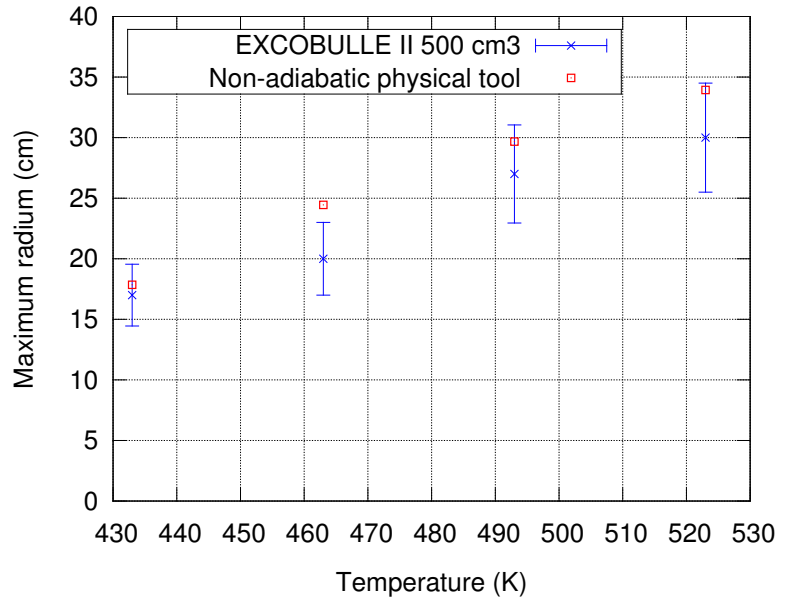

(a)

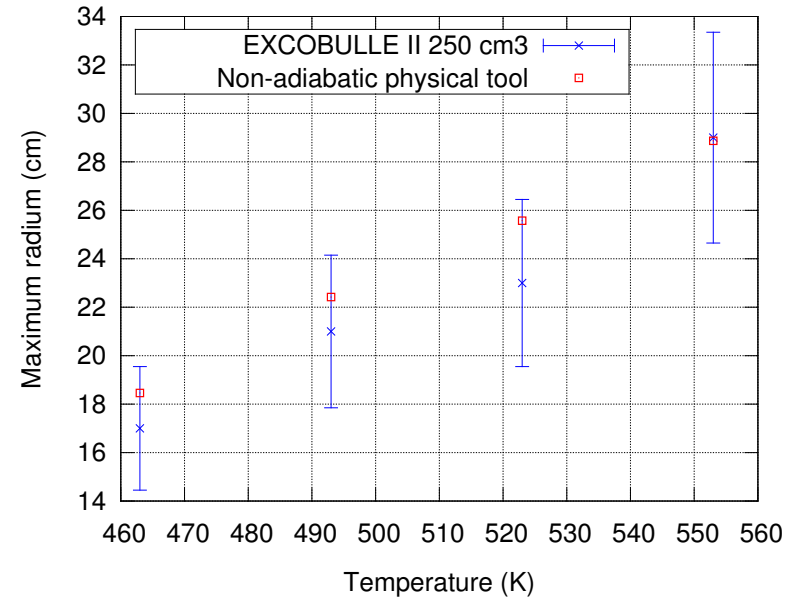

(b)

Figure 6: Hot water source bubble radius versus time computed with the physical tool and recorded in the EXCOBULLE II experiment. (6a): $500 \mathrm{~cm}^{3}$ hot water source. (6b): $250 \mathrm{~cm}^{3}$ hot water source .

Figure $6 \mathrm{a}$ and $6 \mathrm{~b}$ display the computed maximum bubble radiuses for an initial volume of $500 \mathrm{~cm}^{3}$ and $250 \mathrm{~cm}^{3}$ of hot water for several initial temperatures. These results are within the uncertainty bars of the EXCOBULLE II measures. This illustrates the good accuracy of the modeling. The adiabatic modeling, 
however, gives radius higher by $100 \%$ than the EXCOBULLE II tests' ones. Such result was expectable: as the expanding superheated steam water is mixed with cold liquid water, no further water vaporization should occur. Therefore the heat transfer between steam and cold water leads to an energy loss, and therefore the non-adiabatic modeling yields lower radiuses and lower expansion works.

\subsection{Adiabatic modeling comparison to the FUSTIN code}

The FUSTIN code was developed by Chellapandi et al. (2010a,b) to compute Core Disruptive Accidents of Sodium Fast Reactors in the frame of the PFBR development. As FUSTIN's models are different from the physical tool models, the comparison is done on the expansion energy, which should be the same. Indeed, FUSTIN's models also focus on fluid-structure interactions and mechanical phenomena, that are not modeled by the physical tool described in the present paper. However, FUSTIN computes only adiabatic expansions and do not directly models the heat transfer and vaporization phenomena. Instead, those are taken into account in a bubble pressure law $p$ function of bubble volume $V$ written

$p[\mathrm{MPa}]=4\left(\frac{2.56}{V}\right)^{0.72}$.

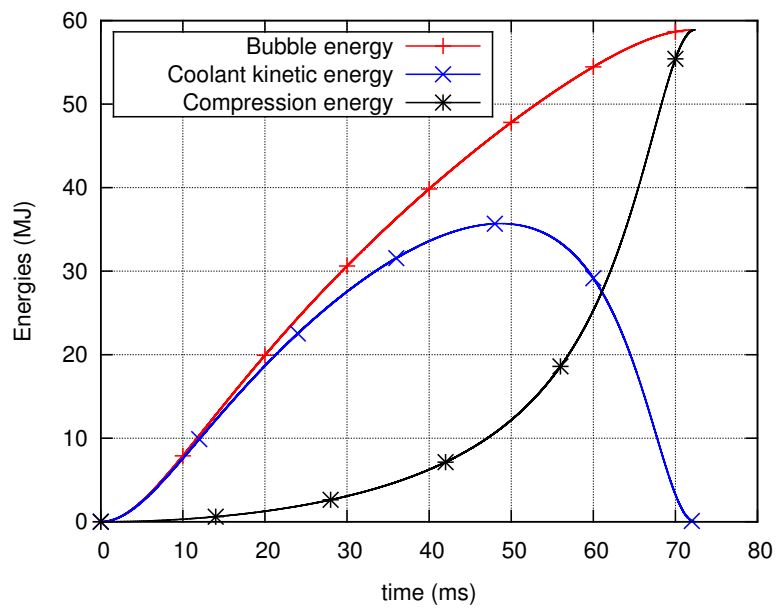

Figure 7: CDA energies in the PFBR geometry

Comparing the physical tool to the FUSTIN code is interesting because it allows to assess the adiabatic expansion modeling. Moreover, FUSTIN has been validated on a large reactor-sized geometry, which is the kind of geometry that the physical tool aims to model. Adiabatic physical tool calculations are conducted on the PFBR geometry with the the pressure law (35). The calculations are based on the adiabatic modeling described in section 2.2. The total energy of the fuel bubble $W_{f}^{v}$, the sodium kinetic energy $K_{N a}$ (demonstrated in appendix A) and the cover gas compression energy $W_{A r}$ are evaluated by the physical tool as

$$
\begin{aligned}
W_{f}^{v}(t) & =\int_{t=0}^{t} p_{f}^{v} 4 \pi\left(R_{f}^{v}\right)^{2} \dot{R}_{f}^{v} \mathrm{~d} t, \\
K_{N a}(t) & =2 \rho_{N a} \pi\left(R_{f}^{v}\right)^{4}\left(\dot{R}_{f}^{v}\right)^{2}\left(\frac{1}{R_{f}^{v}}-\frac{1}{R_{N a}^{l}}\right), \\
W_{A r}(t) & =\int_{t=0}^{t} p_{A r} 4 \pi\left(R_{f}^{v}\right)^{2} \dot{R}_{f}^{v} \mathrm{~d} t .
\end{aligned}
$$

The energies computed by the physical tools are displayed in figure 7. The calculation stops when the fuel vapor expansion velocity reaches zero. The bubble energy is of $59 \mathrm{MJ}$ after an expansion duration of $72 \mathrm{~ms}$. 
Chellapandi et al. (2010b) find using FUSTIN a bubble energy of $61 \mathrm{MJ}$, which is very close of the physical model's result. This result is satisfactory, because it shows that even without modeling complex mechanical phenomena, the total energy found by the fast-running tool is close to a more advanced code.

The conclusion of sections 3.1 and 3.2 is that the physical tool yields decent results given its simplicity. The bubble energy is of the good order of magnitude. However, the reactor results should be treated carefully because not accounting for the vessel mechanical deformation leads to wrong expansion times. The physical tool could therefore be improved by implementing mechanical strain laws. Considering the low calculation times, the modeling yields acceptable results and the modeling is validated.

\section{Physical tool calculations}

Once the tool is validated (section 3), tests are conducted in order to assess the tool results' variability and to provide insights for reactor study. The computations done in section 4.1 do not account for radiative heat transfer. In section 4.2, radiative heat transfer is modeled and a sensitivity analysis is performed on its intensity. The geometry and initial conditions used in the following computations, given in table 1 , are typical of a power SFR reactor.

Table 1: Calculations initial conditions

\begin{tabular}{lccc}
\hline Fuel temperature $[\mathbf{K}]$ & $\mathbf{3 8 0 0}$ & $\mathbf{4 0 0 0}$ & $\mathbf{4 5 0 0}$ \\
\hline Vessel radius $[\mathbf{m}]$ & 7 & 7 & 7 \\
Argon volume $\left[\mathbf{m}^{3}\right]$ & 196 & 196 & 196 \\
Fuel saturation pressure $[\mathbf{M P a}]$ & 0.2 & 0.5 & 3.1 \\
Vapor fuel density $\left[\mathrm{kg} \cdot \mathbf{m}^{-3}\right]$ & 0.8 & 1.6 & 5.7 \\
Sodium mass $[\mathrm{kg}]$ & $1.10^{6}$ & $1.10^{6}$ & $1.10^{6}$ \\
Sodium temperature $[\mathbf{K}]$ & 830 & 830 & 830 \\
\hline
\end{tabular}

\subsection{Entrainment models comparisons for various initial conditions}

Bubble energies computed for each modeling with equation (36) for an initial mass of $5000 \mathrm{~kg}$ heated at $3800 \mathrm{~K}$ are displayed in figure 8. Their final values are below $25 \mathrm{MJ}$, which is quite low because most SFR are designed to withstand a several hundred of MegaJoules release (Bertrand et al., 2015). This was expectable because the fuel pressure saturation pressure at $3800 \mathrm{~K}$ is low, close to $0.2 \mathrm{MPa}$. In this case, the adiabatic modeling releases an energy higher by $20 \%$ than the non-adiabatic one. Indeed, in this case the heat transfer is not high enough to vaporize some sodium in $300 \mathrm{~ms}$, but as this heat transfer occurs, it decreases the expansion energy. Yet, for such a low value, this difference is not very meaningful.

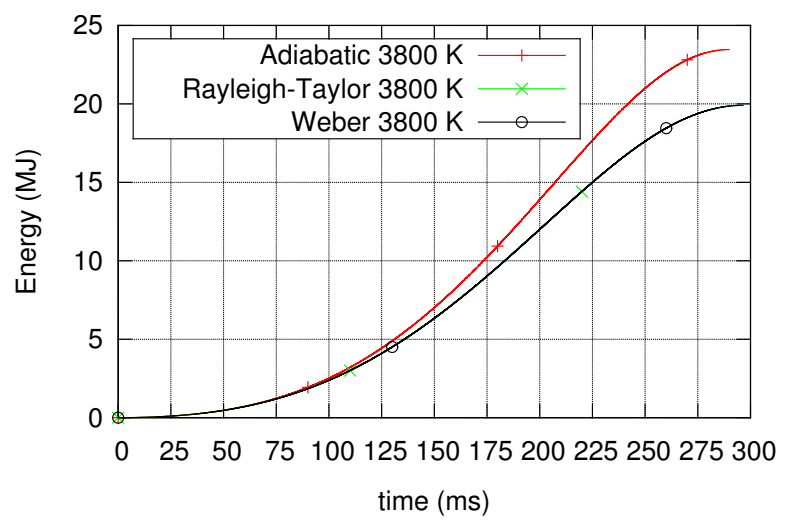

Figure 8: Energy releases for a $(5000 \mathrm{~kg}, 3800 \mathrm{~K})$ initial condition computed with each entrainment modeling. 


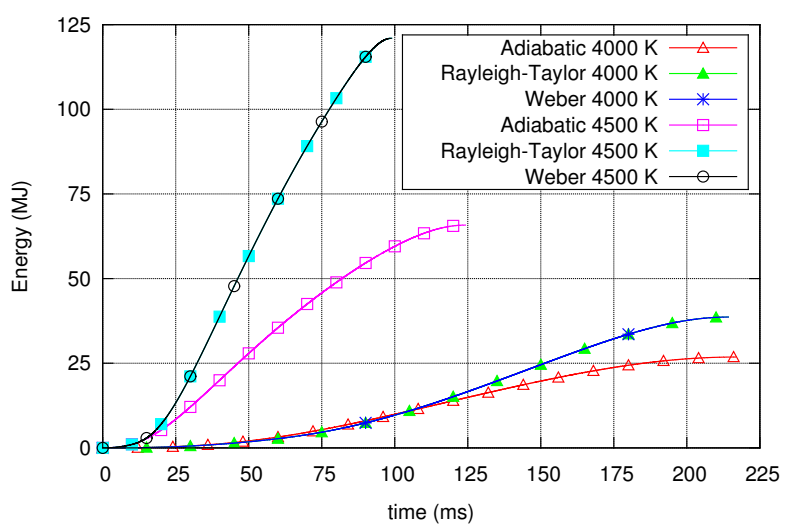

(a)

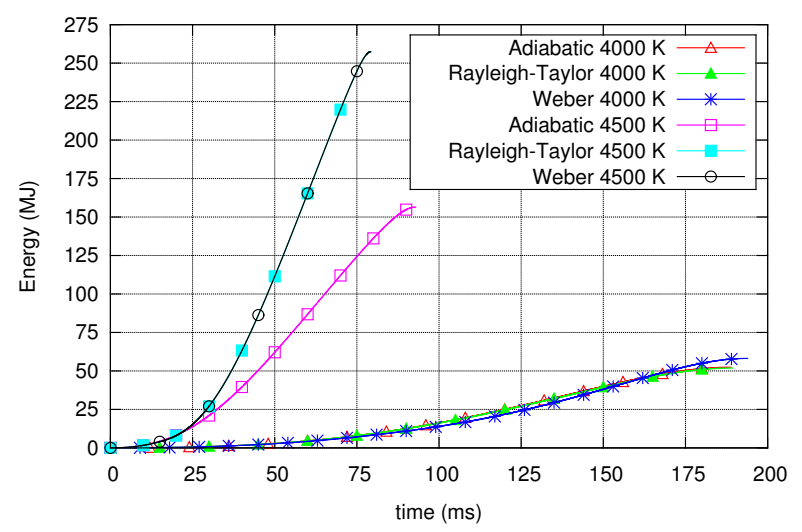

(b)

Figure 9: Energy releases computed with both entrainment modeling at $4000 \mathrm{~K}$ and $4500 \mathrm{~K}$. (9a): Initial fuel mass of $1000 \mathrm{~kg}$. (9b): Initial fuel mass of $5000 \mathrm{~kg}$.

Figures 9a and 9b display energy releases for initial masses of $1000 \mathrm{~kg}$ and $5000 \mathrm{~kg}$ at $4000 \mathrm{~K}$ and $4500 \mathrm{~K}$. At $4000 \mathrm{~K}$, the adiabatic and non-adiabatic models lead to similar results. In this case, the sodium vaporization is just sufficient to compensate the fuel condensation. At $4500 \mathrm{~K}$, the non-adiabatic modeling releases an energy higher by $60 \%$ than the adiabatic modeling. The increase of initial fuel mass also increases the released energy. This mass increase implies a thermal inertia increase, so the fuel temperature decreases slower and therefore the fuel pressure remains high for a longer time, hence leading to a mechanical work increase.

In all these case, the Weber modeling displays the same results than the Rayleigh-Taylor modeling. These calculations show that the transient is very dependent of the initial fuel temperature. Moreover, when the fuel temperature is high enough, the effect of the fuel vapor/liquid sodium heat transfer is not negligible. Note also from figure 9 that the computed expansion times are consistent with the characteristic expansion time estimated with dimensional analysis in section 2.2.3, of the order of $100 \mathrm{~ms}$.

The results of the $(5000 \mathrm{~kg}, 4500 \mathrm{~K})$ expansion in the end of the transient are displayed in table 2 . It shows that the impulse is also higher by $20 \%$ for the non-adiabatic expansion, because the cover gas pressure increases faster. This faster cover gas pressure increase is also the reason why the non-adiabatic computation ends earlier, as it is blocked by the compressed gas earlier. The computations' real times are inferior to two minutes, which is satisfactory ${ }^{1}$.

The conclusion here is that the non-adiabatic modeling should be used preferentially than the adiabatic one, as it accounts for initial condition's variability with more accuracy. The Weber modeling gave the same results than the Rayleigh-Taylor modeling because the fuel vapor inertia is not high enough to break the Rayleigh-Taylor-sized sodium droplets. It might be more important at higher temperatures because the vapor density increases with temperature, but other phenomena would be expected in this case.

\subsection{Radiative heat transfer and Rayleigh-Taylor wavelength influence}

The studies done in previous sections assume that the fuel vapor/liquid sodium heat transfer is only convective. The present section is dedicated to the study of the radiative heat transfer influence. As a simple approach, it is proposed to use the simple grey-body radiative heat transfer law defined by equation (28) and to vary the sodium emissivity $\varepsilon_{N a}$. Barnett et al. (1985) proposed an emissivity correlation for liquid sodium (equation (59) in appendix B) that gives $\varepsilon_{N a} \approx 0.05$ at $800 \mathrm{~K}$. The SIMMER numerical

\footnotetext{
${ }^{1}$ The computations were done on a personal computer with an eight-core Intel ${ }^{\circledR}$ Xeon ${ }^{\circledR}$ E3-1240 of 3.40 GHz and 32 Go of RAM using the MATLAB ${ }^{\circledR}$ software.
} 
Table 2: Final parameters of interest of adiabatic and non-adiabatic computations for a $(5000 \mathrm{~kg}, 4500 \mathrm{~K})$ initial condition

\begin{tabular}{ccc}
\hline Modeling & Adiabatic & Rayleigh-Taylor \\
\hline Expansion time (ms) & 93 & 79 \\
Vapor radius (m) & 3.5 & 3.6 \\
Energy (MJ) & 160 & 260 \\
Impulse (Pa.s) & $6.7 .10^{4}$ & $8.2 .10^{4}$ \\
Fuel Vapor mass (kg) & 300 & 150 \\
Fuel temperature (K) & 4000 & 3800 \\
Bubble pressure (MPa) & 0.5 & 0.9 \\
Cover gas pressure (MPa) & 9 & 27 \\
Sodium vapor mass (kg) & - & 36 \\
Real time calculation (s) & 32 & 96 \\
\hline
\end{tabular}

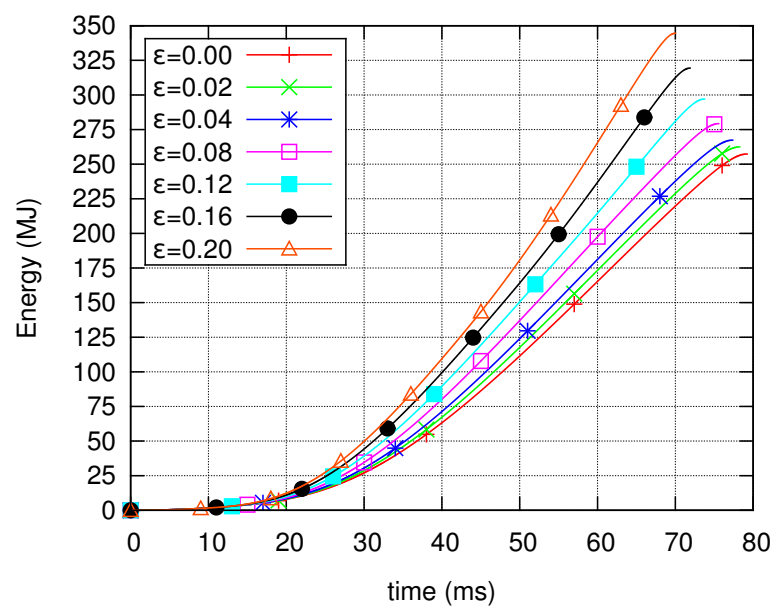

(a)

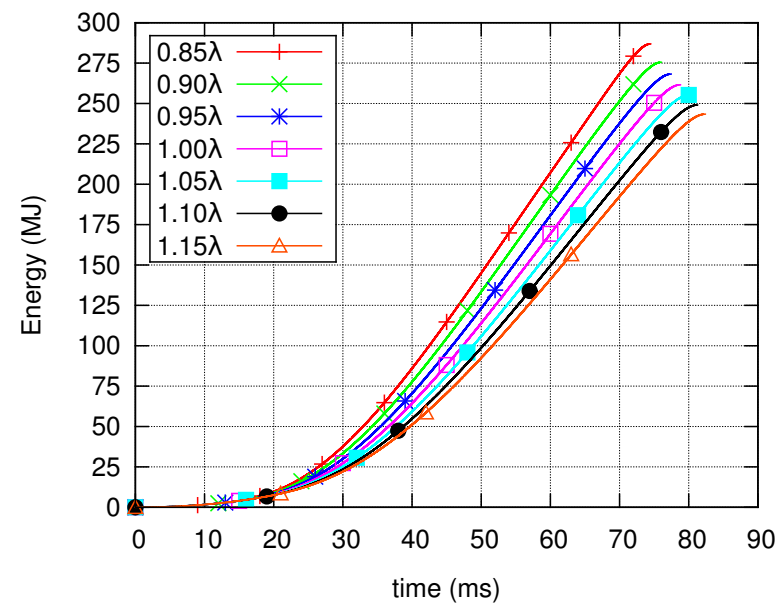

(b)

Figure 10: Energy releases over time for a $(5000 \mathrm{~kg}, 4500 \mathrm{~K})$ initial condition. (10a): Variable liquid sodium emissivity. (10b): Variable critical Rayleigh-Taylor wavelength.

tool uses a much higher constant value of 0.2 . Computations are thus done for a sodium emissivity ranging from 0 to 0.2 . The computations are conducted for an initial fuel mass of $5000 \mathrm{~kg}$ at $4500 \mathrm{~K}$ with the Rayleigh-Taylor entrainment modeling. The results presented in figure 10a show some discrepancies, with an energy release higher by $36 \%$ for the 0.2 -emissivity upper case than for the zero-emissivity lower case.

Similarly, a parametric study is conducted upon the Rayleigh-Taylor wavelength. Assuming that the instability occurs only for a wavelength equal to $\lambda_{R T}^{M}$ might be too restrictive, so computations are conducted with a wavelength ranging from $0.85 \lambda_{R T}^{M}$ to $1.15 \lambda_{R T}^{M}$. The corresponding final energy presented in figure 10b shows discrepancies of $15 \%$, the higher energy being obtained for the smaller wavelength.

Hence, the tool is more sensitive to heat transfer variations than to Rayleigh-Taylor wavelength variations, while both have an influence on results. Predicting the droplets' size with accuracy is therefore important, but the radiative heat transfer modeling seems more critical. The Weber stability criterion does not bring a change of results, because the sodium droplets are stable enough in this range of temperature.

\section{Conclusion and prospects}

In this paper, a modeling of the expansion stage of a Core Disruptive Accident is presented. The transient modeled involves the vaporization of molten fuel, previously superheated by a power excursion, 
and the mechanical loading of the reactor vessel due to vapors expansion. The modeling is designed to provide results with short calculation times in order to be able to perform parametric studies and sensitivity analyses. The associated tool assumes a certain number of simplifying hypotheses that are justified using dimensional analysis and characteristic times comparison.

The tool ensures computation times around one minute. It models the fuel vaporization in a spherical geometry with several options, either adiabatic or non-adiabatic. The adiabatic model considers that the reactor sodium coolant is a liquid piston at constant temperature. The non-adiabatic modeling considers a heat transfer between the fuel vapor and the liquid sodium being entrained into the fuel vapor bubble by a Rayleigh-Taylor instability. A droplet Weber stability criterion is also considered. The tool models the heat transfer as a forced convection accounting for fuel condensation, but it can be coupled to a grey-body radiative heat transfer model.

The tool was validated on the EXCOBULLE II tests at the experiment scale. The non-adiabatic modeling results are within the experimental uncertainty bars. The tool was also validated on a reactor-sized geometry with FUSTIN calculations, an other tool dedicated to such transient and based on an adiabatic expansion hypothesis. In term of released energy and vessel mechanical impulse, both tools give comparable results.

Parametric computations were done for various initial masses and temperatures (section 4.1). The tendency highlighted is that non-adiabatic computations generate more mechanical energy than adiabatic ones, except for low initial fuel temperatures yielding similar energies. As the non-adiabatic modeling was validated on the EXCOBULLE II tests and as it leads to more penalizing energies, it would therefore be adequate to consider it for further safety studies. The Weber stability criterion does not bring a lot of improvement to the Rayleigh-Taylor modeling.

A sensitivity analysis is conducted on the heat transfer and Rayleigh-Taylor wavelength. It appears that the tool is sensitive to these two parameters, the tool being more sensitive to the radiative heat transfer. Investigating the variability associated to these two parameters with statistical techniques is therefore required.

To conclude, the analysis conducted in this paper enlightens two major phenomena (fragmentation and heat transfer) that must be studied accurately to model a fuel vaporization-expansion transient. These parameters are not the only ones: the materials thermo-physical data are also sources of uncertainty. The fuel pressure law, especially, is important because it governs the expansion.

It should also be stated that if the fuel temperature is high enough, the fuel vaporization might lead to the burst of the molten pool, triggering the thrust of hot liquid fuel droplets into the cold liquid sodium. This is a fuel-coolant interaction scenario, and the mechanical energy release would be different in such situation. Therefore, the modeling should be improved in order to treat such fuel burst-vaporization as well as the associated fuel-coolant interaction. In this case, it would also be required to model the mixed vapor behavior when the sodium vapor mass becomes higher than the fuel vapor mass.

\section{Acknowledgement}

This research is conducted with a financial support from the R4G program at CEA and the R\&D division of EDF, which are gratefully acknowledged. Discussion with Dr. Ruyer from IRSN Cadarache was greatly appreciated.

\section{Bibliography}

Abramzon, B. and Sirignano, W. A. (1989). Droplet vaporization model for spray combustion calculations. International Journal of Heat and Mass Transfer, 32(9):1605-1618.

Barnett, P. G., Gentry, P. J., Jackson, J. D., and Tong, D. K. W. (1985). Emissivity measurements of liquid sodium and some sodium contaminated steel surfaces. Proceedings of the IAEA International Working Group Meeting on Heat and Mass Transfer in Fast Reactors, Report IWGFR-5\%, pages 8-10. AERE, Harwell.

Bergman, T. H., Lavine, A. S., Incropera, F. P., and Dewitt, D. P. (2012). Fundamentals of Heat and Mass Transfer. John Wiley and Son, $7^{\text {th }}$ edition.

Berthoud, G., Amblard, M., and Malterre, P. (1984). Expansion and collapse of large bubbles of hot two-phase water in cold water: the Excobulle program. ASME Heat Transfer Conference. Chicago. 
Bertrand, F., Marie, N., Prulhière, G., Lecerf, J., and Seiler, J.-M. (2015). Comparison of the behaviour of two core designs for ASTRID in case of severe accidents. Nuclear Engineering and Design.

Bethe, H. A. and Tait, J. H. (1956). An estimate of the order of magnitude of the explosion when the core of a fast reactor collapses. RHM.

Brennen, C. E. (1995). Cavitation and Bubble Dynamics. Oxford University Press.

Chellapandi, P., Chetal, S. C., and Raj, B. (2010a). Structural integrity assessment of reactor assembly components of a pooltype sodium fast reactor in a core disruptive accident-I: Development of computer code and validations. Nuclear Technology, 172(1):1-15

Chellapandi, P., Chetal, S. C., and Raj, B. (2010b). Structural integrity assessment of reactor assembly components of a pooltype sodium fast reactor in a core disruptive accident-II: Analysis for a 500-MW(electric) prototype fast breeder reactor. Nuclear Technology, 172(1):16-28.

Cho, D. H. and Epstein, M. (1974). Work Potential from a Mechanical Disassembly of the Voided FFTF Core. Technical report ANL-74-17, Argonne National Laboratory.

Cho, D. H., Irvins, R., and Wright, R. (1972). A rate-limited model of moten-fuel/coolant interactions: model development and preliminary calculations. Technical report ANL-79-19, Argonne National Laboratory.

Corradini, M. L. (1978). Heat transfer and fluid flow aspects of fuel-coolant interactions. Report Number COO-2781-12TR, MIT.

Corradini, M. L., Rohsenow, W. M., and Todreas, N. E. (1980). The effects of sodium entrainment and heat transfer with two-phase $\mathrm{UO}_{2}$ during a hypothetical core disruptive accident. Nuclear Science and Engineering, 73(3):242-258.

Delhaye, J.-M. (2008). Thermohydraulique des réacteurs. EDP Science.

Droin, J.-B., Marie, N., Bertrand, F., and Merle-Lucotte, E. (2015). Physico-probabilistic modelling of the primary phase of an Unprotected Loss Of Flow. The $16^{\text {th }}$ International Topical Meeting on Nuclear Reactor Thermalhydraulics, Nureth-16. Chicago, USA, August 30 - September 4.

Epstein, M., Fauske, H. K., Kubo, S., Nakamura, T., and Koyama, K. (2001). Liquid entrainment by an expanding core disruptive accident bubble - a Kelvin-Helmholtz phenomenon. Nuclear Engineering and Design, 210:53-77.

Fermigier, M., Limat, L., Wesfreid, J. E., Boudinet, P., and Quilliet, C. (1992). Two-dimensional patterns in Rayleigh-Taylor instability of a thin layer. Journal of Fluid Mechanics, 236:349-383.

Herbreteau, K., Marie, N., Bertrand, F., Seiler, J.-M., and Rubiolo, P. R. (2016). Analytical modelling of an unprotected transient overpower in sodium-cooled fast reactors. The Nuclear Materials Conference, NuMat2016. Montpellier, France, November 7-10.

IAEA (2008). Thermophysical Properties of Materials For Nuclear Engineering: A Tutorial and Collection of Data. International Atomic Energy Agency, Vienna.

Ishii, M. and Hibiki, T. (2011). Thermo-Fluid Dynamics of Two-Phase Flow. Springer-Verlag New York.

Jacobs, J. W. and Catton, I. (1988). Three-dimensional Rayleigh-Taylor instability Part 1. Weakly nonlinear theory. Journal of fluid mechanics, 187:329-352.

Kolev, N. I. (2012). Multiphase Flow Dynamics 2. Mechanical Interactions. Springer-Verlag Berlin Heidelberg.

Labuntzov, D. A., Lolchugin, B. A., Golovin, V. S., Zakharova, Z. A., and Vladimirova, L. N. (1964). High speed camera investigation of bubble growth for saturated water boiling in a wide range of pressure variations. Thermophysics of High Temperature, 2:446-453.

Lee, K. and Riley, D. J. (1966). The Evaporation of Water Droplets in Superheated Steam. Journal of Heat Transfer.

Lewis, D. J. (1950). The Instability of Liquid Surfaces when Accelerated in a Direction Perpendicular to their Planes. II. Proceedings of the Royal Society of London A: Mathematical, Physical and Engineering Sciences, 202(1068):81-96.

Marie, N., Bachrata, A., Seiler, J.-M., Barjot, F., Marrel, A., Gossé, S., and Bertrand, F. (2016a). A physical tool for severe accident mitigation studies. Nuclear Engineering and Design, 309:224-235.

Marie, N., Marrel, A., Seiler, J.-M., and Bertrand, F. (2016b). Physico-statistical approach to assess the core damage variability due to a total instantaneous blockage of SFR fuel sub-assembly. Nuclear Engineering and Design, 297:343-353.

Marie, N., Marrel, A., Seiler, J.-M., Pérot, N., and Bertrand, F. (2013). Analytical assessment of a total instantaneous blockage of a fuel sub-assembly (TIB) in a sodium fast reactor and its uncertainty analysis. The 15 ${ }^{\text {th }}$ International Topical Meeting on Nuclear Reactor Thermalhydraulics, Nureth-15. Pisa, Italy, May 12-16.

Marrel, A., Marie, N., and De Lozzo, M. (2015). Advanced surrogate model and sensitivity analysis methods for sodium fast reactor accident assessment. Reliability Engineering and System Safety, 138:232-241.

Morita, K. and Fischer, E. A. (1998). Thermodynamic properties and equations of state for fast reactor safety analysis: Part I: Analytic equation-of-state model. Nuclear Engineering and Design, 183(3):177-191.

Morita, K., Fischer, E. A., and Thurnay, K. (1998). Thermodynamic properties and equations of state for fast reactor safety analysis: Part II: Properties of fast reactor materials. Nuclear Engineering and Design, 183(3):193-211.

Petrescu, S. (1994). Comments on "The optimal spacing of parallel plates cooled by forced convection". International Journal of Heat and Mass Transfer, 37:1238.

Pinhasi, G. A., Ullmann, A., and Dayan, A. (2007). 1D plane numerical model for boiling liquid expanding vapor explosion (BLEVE). International Journal of Heat and Mass Transfer, 50(23):4780-4795.

Reynolds, A. B. and Berthoud, G. (1981). Analysis of Excobulle two-phase expansion tests. Nuclear Engineering and Design, $67(1): 83-100$.

Risse, B. (2012). Continuous crystallization of ultra-fine energetic particles by the Flash-Evaporation Process. PhD thesis, Université de Lorraine.

Sharp, D. H. (1984). An overview of Rayleigh-Taylor Instability. Physica 12D, pages 3-18.

Taylor, G. (1950). The Instability of Liquid Surfaces when Accelerated in a Direction Perpendicular to their Planes. I. 
Proceedings of the Royal Society of London A: Mathematical, Physical and Engineering Sciences, 201(1065):192-196.

Villermaux, E. (2007). Fragmentation. Annual Reviews of Fluid Mechanics, 39:419-446.

Wagner, W. and Pruß, A. (2002). The IAPWS formulation 1995 for the thermodynamic properties of ordinary water substance for general and scientific use. Journal of physical and chemical reference data, 31(2):387-535.

Zuber, N. (1959). Hydrodynamics aspects of boiling heat transfer. PhD thesis, University of California, Los Angeles.

\section{Appendix A Equations demonstration}

\section{A.1 Sodium momentum balance}

The Rayleigh equation demonstration is slightly different from the classical one that can be found in most textbooks, because here the inertial liquid has an external boundary. Neglecting gravity, viscosity and surface tension, the momentum balance equation of sodium reads

$\rho_{N a}^{l} \frac{\mathrm{d} \vec{v}}{\mathrm{~d} t}+\rho_{N a}^{l} \vec{v} \cdot \vec{\nabla}[\vec{v}]=-\vec{\nabla}[p]$

Assuming a purely radial motion, the continuity equation is $\vec{\nabla} \cdot \vec{v}=0$. Sodium velocity $v$ therefore reads $v=f(t) / r^{2}$, where $f(t)$ is only function of time. Velocity at fuel bubble boundary gives $f(t)=\left(R_{f}^{v}\right)^{2} \dot{R}_{f}^{v}=$ $\left(R_{N a}^{l}\right)^{2} \dot{R}_{N a}^{l}$. Hence, integrating equation (39) over the sodium phase leads to the Rayleigh equation

$R_{f}^{v} \ddot{R}_{f}^{v}-R_{N a}^{l} \ddot{R}_{N a}^{l}+\frac{3}{2}\left(\dot{R}_{f}^{v}\right)^{2}-\frac{3}{2}\left(\dot{R}_{N a}^{l}\right)^{2}=\frac{p_{f}^{v}-p_{A r}}{\rho_{N a}^{l}}$.

This latest yields the differential equation (13).

\section{A.2 Two-phase fuel enthalpy balance}

The enthalpy balance of the two-phase fuel liquid-vapor system is (Delhaye, 2008)

$\frac{\mathrm{d} H_{f}^{l+v}}{\mathrm{~d} t}=\frac{\delta W}{\mathrm{~d} t}-\frac{\delta Q^{t o t}}{\mathrm{~d} t}+\frac{\mathrm{d}\left(p_{f} V_{f}^{l+v}\right)}{\mathrm{d} t}$.

Where $\delta Q^{\text {tot }}$ is the total heat transferred to the sodium. It is either null in the adiabatic case or defined by equation (32). The fuel work is $\delta W=-p_{f}^{v} \mathrm{~d} V_{f}^{v}$. Therefore equation (41) becomes

$\frac{\mathrm{d} H_{f}^{l+v}}{\mathrm{~d} t}=V_{f}^{l+v} \frac{\mathrm{d} p_{f}^{v}}{\mathrm{~d} t}-\frac{\delta Q^{t o t}}{\mathrm{~d} t}$.

The two-phase fuel enthalpy is expressed as a function of fuel vapor and liquid fuel enthalpies

$H_{f}^{l+v}=m_{f}^{l+v}\left(x h_{f}^{v}+(1-x) h_{f}^{l}\right)$,

where $x=m_{f}^{v} / m_{f}^{l+v}$ is the fuel vapor quality. This allows to write the two-phase fuel enthalpy variation as

$\frac{\mathrm{d} H_{f}^{l+v}}{\mathrm{~d} t}=m_{f}^{l+v}\left(x \frac{\mathrm{d} h_{f}^{v}}{\mathrm{~d} t}+(1-x) \frac{\mathrm{d} h_{f}^{l}}{\mathrm{~d} t}+h_{f}^{l+v} \frac{\mathrm{d} x}{\mathrm{~d} t}\right)$.

Near saturation conditions, enthalpies are only function of temperature, so

$\frac{\mathrm{d} H_{f}^{l+v}}{\mathrm{~d} t}=m_{f}^{l+v}\left\{\left(x \frac{\mathrm{d} h_{f}^{v}}{\mathrm{~d} T}+(1-x) \frac{\mathrm{d} h_{f}^{l}}{\mathrm{~d} T}\right) \frac{\mathrm{d} T}{\mathrm{~d} t}+h_{f}^{l+v} \frac{\mathrm{d} x}{\mathrm{~d} t}-\frac{\delta Q^{t o t}}{\mathrm{~d} t}\right\}$.

Combining equations (42) and (45) leads to equation (16) for the adiabatic case and to equation (34) for the non-adiabatic one. 


\section{A.3 Two-phase fuel mass balance}

Likewise, the two-phase fuel mass is written as the combination

$V_{f}^{l+v}=m_{f}^{l+v}\left(\frac{x}{\rho_{f}^{v}}+\frac{1-x}{\rho_{f}^{l}}\right)$.

A time derivation yields

$\frac{\mathrm{d} V_{f}^{l+v}}{\mathrm{~d} t}=m_{f}^{l+v}\left\{\left(\frac{1}{\rho_{f}^{v}}-\frac{1}{\rho_{f}^{l}}\right) \frac{\mathrm{d} x}{\mathrm{~d} t}+\left(x \frac{\mathrm{d}\left(1 / \rho_{f}^{v}\right)}{\mathrm{d} t}+(1-x) \frac{\mathrm{d}\left(1 / \rho_{f}^{l}\right)}{\mathrm{d} t}\right)\right\}$.

Near saturation line, fuel densities are only functions of temperature. Similarly than for equation (16), fuel densities' derivatives versus time can be expanded as derivatives versus temperature, yielding equation (18).

\section{A.4 Sodium instantaneous global kinetic energy}

The liquid sodium phase integral kinetic energy is

$K_{N a}=\int_{V_{N a}} \frac{\rho_{N a}^{l} v_{N a}^{2}}{2} \mathrm{~d} V=\int_{r=R_{f}^{v}}^{r=R_{N a}^{l}} \frac{\rho_{N a}^{l}\left(v_{N a}(r)\right)^{2}}{2} 4 \pi r^{2} \mathrm{~d} r$.

If the sodium sodium is incompressible, its local velocity reads $v_{N a}(r)=f(t) / r^{2}$ (section A.1). The integral (48) is therefore

$K_{N a}=2 \pi \rho_{N a}^{l}(f(t))^{2} \int_{r=R_{f}^{v}}^{r=R_{N a}^{l}} \frac{\mathrm{d} r}{r^{2}}=2 \pi \rho_{N a}^{l}(f(t))^{2}\left(\frac{1}{R_{f}^{v}}-\frac{1}{R_{N a}^{l}}\right)$.

Using the expression $f(t)=\left(R_{f}^{v}\right)^{2} \dot{R}_{f}^{v}$, the latter yields

$K_{N a}=2 \pi \rho_{N a}^{l}\left(R_{f}^{v}\right)^{4}\left(\dot{R}_{f}^{v}\right)^{2}\left(\frac{1}{R_{f}^{v}}-\frac{1}{R_{N a}^{l}}\right)$.

\section{A.5 Sodium droplets flat temperature profile}

Equation (29) is written under the assumption that the temperature profile inside the bubble is flat, requiring that the heat transfer inside the bubble is faster than the heat transfer outside the bubble. To prove that equation (29) can be applied, the heat internal and external droplet heat transfer coefficients are compared. A shear flow applied to a liquid drop generates a Hill's vortex internal convection motion (figure 11). The corresponding internal heat transfer is evaluated as a function of an effective thermal conductivity $k_{N a}^{\text {eff }}$, obtained with a correlation established by Abramzon and Sirignano (1989) for gasoline sprays

$\frac{k_{N a}^{\mathrm{eff}}}{k_{N a}^{l}}=1.86+0.86 \tanh \left(2.245 \log _{10}\left(\frac{P e}{30}\right)\right)$,

$P e=\frac{0.397 v_{j} d_{j}}{k_{N a}^{l} /\left(\rho_{N a}^{l} c_{p, N a}^{l}\right)} \frac{\eta_{f}^{v}}{\eta_{N a}^{l}}\left(R e_{f}^{v}\right)^{1 / 3}$,

$R e_{f}^{v}=\frac{\rho_{f}^{v} v_{j} d_{j}}{\eta_{f}^{v}}$.

An effective Biot number, comparing the external heat transfer coefficient $\kappa_{j}$ with the internal heat conduction coefficient $k_{N a}^{\mathrm{eff}} / d_{j}$, is built as $B i=\kappa_{j} d_{j} / k_{N a}^{\mathrm{eff}}$. Its value, around 0.1 , shows that the internal heat transfer is faster than the external one. Therefore, it is acceptable to assume that the temperature profile in the liquid droplet is flat, and writing equation (29) is adequate. 


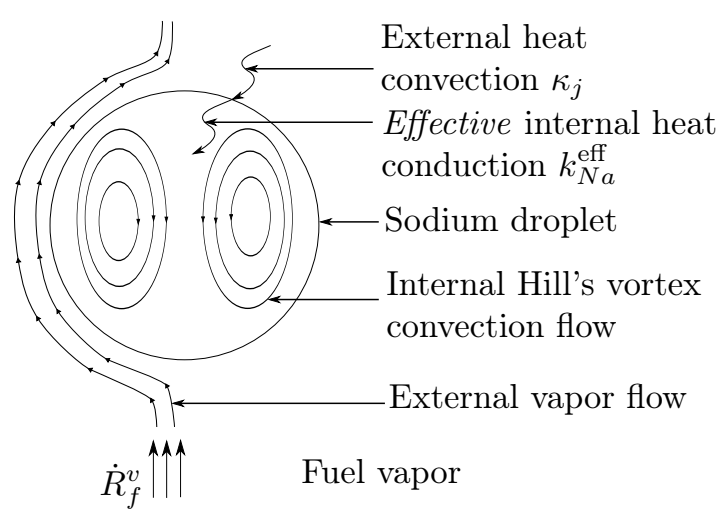

Figure 11: Hill's vortex convective spherical patterns.

\section{Appendix B Thermophysical properties}

\section{B.1 Fuel thermophysical properties}

The two-phase fuel saturation pressure law is described in Bertrand et al. (2015) as

$p_{f}^{\mathrm{sat}}\left(T_{f}\right)[\mathrm{MPa}]=210 \exp \left(6.4\left(1-\frac{7800}{T_{f}[\mathrm{~K}]}\right)\right)$.

The liquid fuel heat capacity is given by the IAEA (2008) as

$\frac{\mathrm{d} h_{f}^{l}}{\mathrm{~d} T}\left[\mathrm{~J}_{\mathrm{kgg}}{ }^{-1} \cdot \mathrm{K}^{-1}\right]=c_{p, f}^{l}=0.93087+\frac{4.921 .10^{9}}{T^{2}\left[\mathrm{~K}^{2}\right]}$.

Data on mixed oxides fuel vapor heat capacity are scarce. A fixed approximative value of $200 \mathrm{~J} \cdot \mathrm{kg}^{-1} \cdot \mathrm{K}^{-1}$ is taken for $\mathrm{d} h_{f}^{v} / \mathrm{d} T$. Liquid fuel density's variations with temperature are evaluated using a correlation given by the IAEA (2008) for uranium dioxide, reading

$\rho_{f}^{l}\left[\mathrm{~kg} \cdot \mathrm{m}^{-3}\right]=8860-0.92852(T[\mathrm{~K}]-3120)$,

$\frac{\mathrm{d}\left(1 / \rho_{f}^{l}\right)}{\mathrm{d} T}=\frac{0.9285}{(8860-0.9285(T[\mathrm{~K}]-3120))^{2}}$.

The fuel vapor thermophysical data are given by Morita and Fischer (1998); Morita et al. (1998)

$\rho_{f}^{v}\left[\mathrm{~kg} \cdot \mathrm{m}^{-3}\right]=1560\left\{\exp \left(B_{G 1} \theta^{\frac{1}{3}}+B_{G 2} \theta^{\frac{2}{3}}+B_{G 3} \theta^{\frac{4}{3}}+B_{G 4} \theta^{3}+B_{G 5} \theta^{\frac{37}{6}}+B_{G 6} \theta^{\frac{71}{6}}\right)\right\}^{-1}$,

$\frac{\mathrm{d}\left(1 / \rho_{f}^{v}\right)}{\mathrm{d} T_{f}^{v}}=-\frac{1}{\rho_{f}^{v}}\left(\frac{1}{3} \frac{B_{G 1}}{10600} \theta^{-\frac{2}{3}}+\frac{2}{3} \frac{B_{G 2}}{10600} \theta^{-\frac{1}{3}}+\frac{4}{3} \frac{B_{G 3}}{10600} \theta^{\frac{1}{3}}+\frac{3 B_{G 4}}{10600} \theta^{2}+\frac{37}{6} \frac{B_{G 5}}{10600} \theta^{\frac{31}{6}}+\frac{71}{6} \frac{B_{G 6}}{10600} \theta^{\frac{65}{6}}\right)$,

with

$\theta=1-\frac{T_{f}^{v}[\mathrm{~K}]}{10600}$,

$B_{G 1}=0.390118, B_{G 2}=2.64047, B_{G 3}=1.79946$,

$B_{G 4}=9.17799, B_{G 5}=23.1365, B_{G 6}=60.7538$. 


\section{B.2 Sodium thermophysical properties}

The sodium vapor is heated over its critical temperature $(2500 \mathrm{~K})$, but its pressure is controlled by the fuel saturation pressure, so it remains under the sodium critical pressure (25 $\mathrm{MPa})$. For such condition, the sodium vapor heat capacity $c_{p, N a}^{v}$ can be approximated to $1000 \mathrm{~J} / \mathrm{kg} / \mathrm{K}$.

The evaluation of the sodium partial pressure is done using a modified Redlich-Kwong equation extracted from the SIMMER code. This equation is an improvement of the Van der Waals equation, reading

$p_{N a}=\frac{T_{N a}^{v} r^{N a}}{\left(1+y_{B, v}\right)\left(\left(1 / \rho_{N a}^{v}\right)-a_{v 1}\right)}-\frac{a\left(T_{N a}^{v}\right)}{\left(1 / \rho_{N a}^{v}\right)\left(\left(1 / \rho_{N a}^{v}\right)+a_{v 3}\right)}$,

with

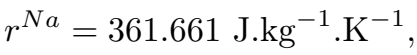

$a_{v 1}=2.93447 .10^{-4}, \quad a_{v 2}=1.23634 .10^{4}$,

$a_{v 3}=1.96134 .10^{-2}, a_{v 4}=4.92937 .10^{-1}$,

$a\left(T_{N a}^{v}\right)=a_{v 2}\left(\frac{T_{N a}^{v}}{T_{N a}^{c r i t}}\right)^{a_{v 4}}, T_{N a}^{v}<T_{N a}^{c r i t}$,

$a\left(T_{N a}^{v}\right)=a_{v 2}\left(1+a_{v 4}\left(\frac{T_{N a}^{v}}{T_{N a}^{c r i t}}-1\right)\right), T_{N a}^{v}>T_{N a}^{c r i t}$,

$y_{B, v}=\frac{1+2 x_{v}-\left(1+8 x_{v}\right)^{1 / 2}}{2\left(x_{v}-a_{v 3}\right)}$,

$x_{v}=\frac{k_{2 v} R_{g p} / r_{N a} T_{N a}^{v}}{\left(1 / \rho_{N a}^{v}\right)-a_{v 1}}$,

$k_{2 v}=\exp \left(d_{v 1}+\frac{d_{v 2}}{T_{N a}^{v}}\right)$,

$d_{v 1}=-21.4845, \quad d_{v 2}=9215.71$.

Barnett et al. (1985) gives the liquid sodium radiative emissivity for temperatures $T_{N a}^{l}$ ranging from $470 \mathrm{~K}$ to $920 \mathrm{~K}$ as

$\varepsilon_{N a}=6.62 .10^{-5} T_{N a}^{l}-6.6826 .10^{-3}$. 